\title{
GENETICS
}

\section{Personalized medicine and tumour heterogeneity}

A team of what Cancer Research UK have described as "some of the UK's most exciting young researchers" have performed a careful genetic analysis of tumours and shown that "no two samples from the same patient were genetically identical." This finding has obvious implications for personalized medicine.

The researchers, led by Charlie Swanton, used multiple samples from four patients with metastatic renal-cell carcinoma that were taken before and after cytoreductive surgery. They used exome sequencing, chromosome aberration analysis and DNA ploidy profiling to study these biopsies. Swanton explains the results: "they describe in some considerable detail the burden of the genetic differences between cancer cells or between regions of one tumour."

This detailed analysis of the biopsies confirmed what has been suspected for some time, no two tumours were the same. Furthermore, no two biopsies of the same tumour were the same. Swanton states "approximately two-thirds of the mutations present in a single biopsy were not shared in all biopsies across the same tumour."

Importantly, these intratumour genetic differences occurred in genes that encode proteins that are targets for some of the available anticancer drugs (such as mTOR-a target of everolimus). These targeted anticancer drugs are the core of personalized therapy and so if mutations in drug targets are only detected in some biopsies but not all, this has obvious implications for the application of personalized medicine. "The main findings of this study illustrate, for us, the complexities of what is known as personalized medicine," says Swanton.

The assessment revealed that the tumours underwent convergent phenotypic evolution with multiple distinct loss of function mutations occurring in the same tumour-suppressor gene across different regions of the tumour. Swanton explains it more clearly: "we are beginning to think of

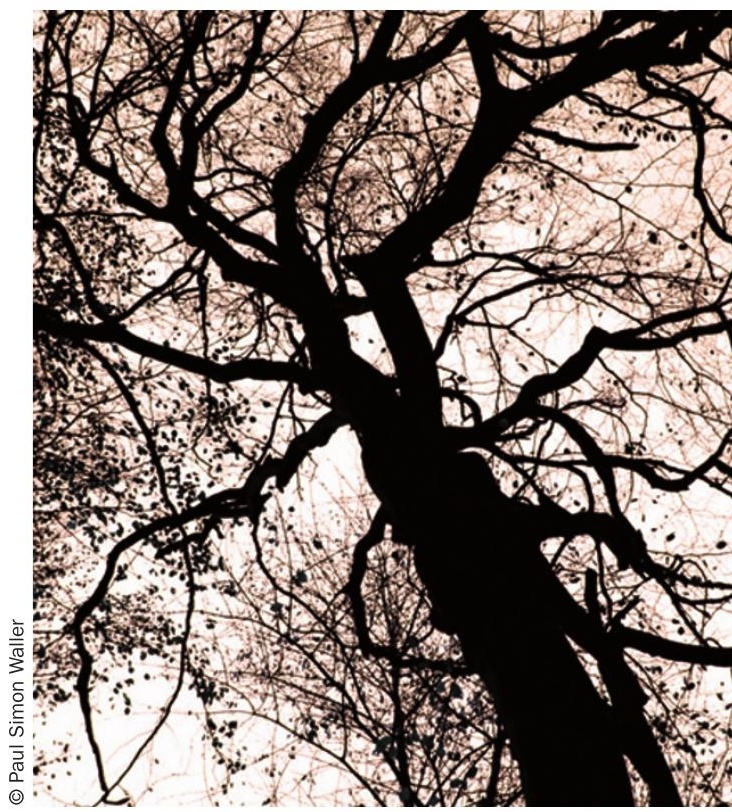

tumours in Darwinian terms, so instead of the concept that we used to have of linear tumour evolution what in fact we're seeing in tumours is a tree-like evolution, similar to what Greaves and Enver demonstrated in haematological malignancies."

Rebecca Kirk

Original article Gerlinger, M. et al. Intratumor heterogeneity and branched evolution revealed by multiregion sequencing. N. Engl. J. Med. 366, 883-892 (2012) Further reading Anderson, K. et al. Genetic variegation of clonal architecture and propagating cells in leukaemia. Nature 469, 356-361 (2011) 